\section{Kinetics of the Decomposition of Molecules of Intermediate Complexity}

IN a large class of gaseous chemical reactions, the mechanism is generally supposed to be the following. Molecules are brought into an activated state by collision. Most of the activated molecules are deactivated again, but during their existence there is a definite probability of their chemical transformation. As is well known, the relation between reaction rate and pressure under these conditions is such that the reciprocal of $\tau$, the half life of the reacting substance, gives a eurve of type 1 or 2 (see Fig. 1) when plotted against the initial pressure ${ }^{1}$. (The precise shape of the curve before it has become horizontal depends upon how the above mentioned probability varies with the total energy of the molecule ${ }^{2}$.)

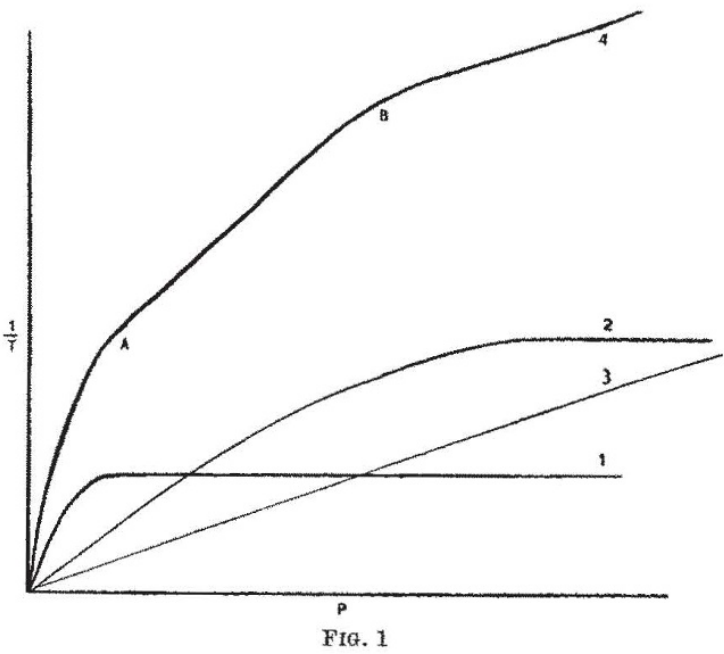

In a molecule of moderate, but not too great, complexity, it seems possible that there may be several different modes of activation, corresponding to particular divisions of part of the energy among a limited number of vibrational (or rotational) states. To a first approximation, each of these modes may be associated with a separate probability of transformation, because, in the absence of collisions, internal redistribution may be difficult. Thus the total rate of reaction will be roughly the sum of several virtually independent reactions, each rate varying according to a curve of the type 1,2, or 3 . Since each of these reaches its limiting rate at a different pressure, the total rate may vary with pressure according to a segmented curve of type 4, with fairly pronounced changes of direction at $A, B$, and so on.

The simplest types of molecule may give curves which do not bend, at least up to high pressures, moderately simple molecules may give curves with a limited number of segments of decreasing slope, while complex molecules with many degrees of freedom give curves in which the segments merge into a single line without any noticeable changes of direction at particular stages.

The equation of the segmented curve in real examples will be very complicated, since the assumption of virtually independent reactions is to some extent an idealisation. Further, the variation of all the transformation probabilities with total energy may also complicate matters.

The object of this note, however, is simply (I) to suggest the desirability of exploring the hypothesis that the most general form of curve for molecules of 'intermediate complexity' is of the segmented type, and (2) to state that experiments on the decomposition of nitrous oxide at fairly low pressures (already published ${ }^{3}$ ) and further experiments on the decomposition of acetaldehyde (in progress) yield indications of the real existence of these different types of activated state.

Balliol and Trinity College

C. N. Hinshelwood. C. J. M. FLetcher.

Laboratory, Oxford. Dec. 8.

"Hinshelwood, "Kinetics".

Rice and Ramsperger, Kassel.
${ }^{3}$ Musgrave and Hinshelwood, Proc. Roy. Soc., A, 135, 23 ; 1932.

\section{'Hexuronic Acid' (Ascorbic Acid) as the Antiscorbutic Factor}

IN view of the facts that (1) hexuronic acid is the name of a class of substances rather than that of one individual compound, and that (2) the material described as hexuronic acid isolated from adrenal cortex and now from Paprika contains a molecule of water less than is required for a hexuronic acid, we wish to ascribe the name ascorbic acid to the crystalline substance $\mathrm{C}_{6} \mathrm{H}_{8} \mathrm{O}_{6}$ which has been the subject of earlier communications from our laboratories.

Universities of Szeged and Birmingham.

A. SZENT-GyörGyt. Dec. 19.

\section{Methylnornarcotine, Glycuronic Acid, and} Vitamin C

THe view that vitamin $\mathrm{C}$ is identical with methyl. nornarcotine has been advanced by $\mathrm{Rygh}^{1,2}$, who claims that narcotine occurs in unripe fruit and disappears with corresponding formation of vitamin $\mathrm{C}$ during ripening. According to Rygh, guinea-pigs receiving a scurvy-producing basal diet together with a suitable daily supplement of synthetic methylnornarcotine lose weight and die but without any of the typical symptoms of seurvy. Other workers ${ }^{3,4,5,6}$ have been unable to confirm this observation or to find narcotine in unripe fruit, and Dalmer and Molls have shown that methylnornarcotine as prepared by Rygh is not a single chemical substance.

In a later communication Rygh ${ }^{7}$ has changed his ground and taken up the position that vitamin $\mathrm{C}$ is a complex, of which one component must be methylnornarcotine while the other may be any uronic acid. Thus according to $\mathrm{Rygh}$, the recently demonstrated anti-scorbutic activity of hexuronic acid is due to the contamination of the hexuronic acid by methylnornarcotine, giving a mixture of the two components necessary for the make-up of vitamin C. This hypothesis was drawn from the observation that guinea-pigs were not only protected from scurvy but also grew normally if in addition to the basal diet they received daily $10 \gamma$ of " 30 per cent methylnomarcotine" and $\frac{1}{2}$ mgm. of glycuronic acid, although the glycuronic acid alone neither prevented scurvy nor supported growth.

I have attempted without success to confirm these 